\title{
Caracterización del comportamiento de las Pymes según el género del gerente: un estudio empírico
}

SME behavior characterization according to the manager's gender: an empirical study

Caractérisation du comportement des PME selon le genre du gérant : une étude empirique

Mónica García Solarte

monica.garcia@correounivalle.edu.co Universidad del Valle Cali - Colombia

Profesora del Departamento de Administración y Organizaciones de la Facultad de Ciencias de la Administración.

Domingo García Pérez de Lema domingo.garcia@upct.es Universidad Politécnica de Cartagena Cartagena - España

Director y catedrático del Departamento de Economía Financiera y Contabilidad.

Antonia Madrid Guijarro antonia.madrid@upct.es Universidad Politécnica de Cartagena Cartagena - España

Profesora Titular del Departamento de Economía Financiera y Contabilidad.

Artículo de investigación científica y tecnológica Según Clasificación Colciencias

Fecha de recepción: $02 / 03 / 2012$

Fecha de corrección: 31/04/2012

Fecha de aprobación: 20/05/2012

\section{Resumen}

El aumento de la participación de la mujer en el mercado laboral ha estimulado que muchas investigaciones incorporen una "perspectiva de género" en el estudio de la actividad empresarial. En este trabajo se ha estudiado cómo el género incide en el comportamiento de una muestra de 600 Pymes de la región de Murcia (España) con base en diferentes variables de caracterización de la empresa. El análisis de los resultados se realiza desde una perspectiva univariante y multivariante a través de una regresión logística. Los resultados revelan que las empresas que son dirigidas por mujeres se encuentran sobre todo en el sector de servicios, son de menor tamaño, tienen una posición tecnológica menos desarrollada, y son mayoritariamente de carácter familiar. Estos resultados vienen a mostrar cómo el ingreso de la mujer al mundo laboral aun hoy se ve influido por el papel que la mujer ha jugado en la sociedad. Por lo tanto, con este trabajo se busca contribuir al desarrollo de estudios de género en la Pyme que permitan identificar procesos de cambio cultural dentro de las organizaciones que se orienten a brindar mayores oportunidades de desarrollo y crecimiento a la mujer.

Palabras clave: género, Pyme, techo de cristal. 


\section{SME behavior characterization according to the manager's gender: an empirical study}

Caracterización del comportamiento de las Pymes según el género del gerente: un estudio empírico

\section{Caractérisation du comportement des}

PME selon le genre du gérant : une étude empirique

\section{Abstract}

Increasing participation of women in the job market has led many researches to incorporate a "gender perspective" within organizational activity studies. This paper studied how gender roles influence the behavior of SMEs in Murcia, Spain, based upon the different variables that characterize the organization by using a sample of 600 different SMEs. The results analysis is made from a univariate and multivariate process through logistic regression. The resulting data reveals that most women-led organizations are found within the service sector, which are smaller, posses less advanced technology, and are family run. This data show how the incorporation of women in the labor world is still influenced by the role women have played in society. Therefore, this paper seeks to contribute to the development of gender studies in SMEs to allow identification of cultural change processes within organizations aimed at providing women with better opportunities.

Keywords: gender, SME's crystal ceiling.

\section{Caractérisation du comportement des PME selon le genre du gérant : une étude empirique}

\section{Caracterización del comportamiento de las Pymes según el género del gerente: un estudio empírico}

\section{SME behavior characterization according to the manager's gender: an empirical study}

\section{Résumée}

Ĺaugmentation de la participation de la femme dans le marché du travail a encouragé beaucoup de recherches à incorporer une "perspective de genre" dans l'étude de l'activité entrepreneuriale. Ce travail porte sur la façon dont le genre met l'accent sur le comportement dans un échantillon de 600 PME de la région de Murcia (Espagne) sur la base de plusieurs variables caractérisant l'entreprise. L'analyse se fait depuis une perspective uni variante et multi variante à travers une régression logistique. Les résultats révèlent que les entreprises dirigées par des femmes se trouvent surtout sur le secteur de services, elles sont de plus petite taille, elles ont une position technologique moins développée, et sont majoritairement à caractère familier. Ces résultats montrent comment l'admission de la femme dans le monde du travail reste toujours aujourd`hui influencée par le rôle que la femme a joué dans la société. Donc, ce travail cherche à contribuer au développement des études de genre dans la PME qui permettent d'identifier des processus de changement culturel dans les organisations orientées à offrir de majeures opportunités de développement et de croissance à la femme.

Mots clef: genre, PME, toit de cristal 


\section{Caracterización del comportamiento de las Pymes según el género del gerente: un estudio empírico}

\section{Introducción}

El ingreso de la mujer al mercado laboral y a las organizaciones en las últimas décadas plantea nuevos interrogantes en torno a la función que ésta desempeña y abre una importante línea de trabajo que estudia el efecto del género en los negocios y las diferencias que se pueden encontrar en las características de las empresas gestionadas por mujeres en relación con las gestionadas por hombres (Sánchez-Apellániz, 1997; Brush, 1992; Bird y Brush, 2002; De Anca y Aragón, 2007; Gallego, García y Rodríguez, 2010; Yordanova, 2011). Esto genera en los académicos un gran interés por la realización de estudios empíricos que identifican cómo la mujer y sus características, impactan en el desarrollo de las organizaciones, en especial en las grandes empresas, donde hoy, se busca un proceso de diversificación, con igualdad de oportunidades y aportes al desarrollo de las prácticas del buen gobierno. Todo se encamina a fomentar un cambio en la gestión corporativa que permita la inserción de la mujer en todos los niveles de la organización.

Estos estudios tienen entre otros objetivos identificar y analizar cómo el género influye en el desempeño de las organizaciones (Escandón y Arias, 2011; Swinney, Runyan y Huddleston, 2006; Talmud y Izreli, 1999), cuál es la percepción y actitud que se tienen hacia las mujeres directivas en las organizaciones y en algunos casos su relación con el desempeño organizacional (López, Gómez y Betancourt, 2011; De Luis, Sánchez, Pérez y Vela, 2007; Lee, 2005), comprobar si hombres y mujeres utilizan diferentes estilos de dirección (Eagly, 2005; Cheung y Halpern, 2010; Ayman y Korabik, 2010), cómo el género afecta el desempeño de las empresas familiares (Danes, Stanfford y Loy, 2007), cómo el género afecta los procesos exportadores de las empresas (Orser, Spence, Riding y Carrington, 2010), y cómo el género afecta el proceso de tomar riesgos, percibir los riesgos o comportarse frente a los riesgos en las empresas (Inanova y Inanova, 2011).

Sin embargo, en las Pymes no se impulsa de la misma manera el desarrollo de prácticas de buen gobierno e igualdad de oportunida- des, y pocos son los estudios realizados en la identificación de los efectos de cómo el género afecta el desempeño de éstas. Una de las pocas investigaciones realizadas es la de Fernández, López, Maeztu y Martín (2010) que tiene como objetivo contribuir al principio de igualdad de oportunidades en el acceso de la mujer a los cargos directivos en las Pymes de la Comunidad Autónoma de Andalucía (España), donde se evidencia que en las Pymes también se presenta la segregación ocupacional vertical, lo que despierta el interés por realizar estudios orientados a portar en el desarrollo de las Pymes y el impacto del género en su actividad.

El objetivo de este artículo es identificar y caracterizar el comportamiento de la Pyme considerando el género del gerente, de acuerdo con factores cómo el tamaño, el sector, el nivel educativo y la posición tecnológica, para así analizar los efectos que tiene el ingreso de la mujer a las organizaciones, y poder identificar las fortalezas que tienen y potencializar el desarrollo de la mujer en la organización. Para ello, se ha desarrollado un estudio empírico con 600 Pymes de la región de Murcia (España), donde se busca contribuir a la literatura desarrollada en el contexto empresarial Pyme español, y al análisis de cómo la incorporación de la mujer en ese entorno requiere de cambios significativos que permitan la igualdad de oportunidades y del desarrollo de prácticas de buen gobierno corporativo.

La estructura del presente documento se divide en cuatro partes: Primero se desarrolla el marco teórico, se revisan los estudios empíricos previos y se plantean las hipótesis a contrastar; luego se expone la metodología donde se define la muestra y la forma de medir las variables; a continuación se presenta el análisis de los resultados del análisis univariante y multivariante realizado; finalmente se extraen las principales conclusiones, y se exponen las limitaciones y futuras líneas de investigación.

\section{Marco teórico y estudios empíricos}

De acuerdo con la Real Academia de la Legua Española se entiende por género a un conjunto 
de seres que tienen una o varias características en común. Lamas (1994) plantea el género como la simbolización cultural construida a partir de la diferencia sexual, desde el nacimiento del ser humano y que se manifiesta en la vida social, política y económica. Entender qué es y cómo opera el género nos ayuda a entender las percepciones específicas sobre las mujeres y los hombres, que se dan dentro de la sociedad y en el ámbito organizacional. Por lo tanto, el "género" o rol sexual, en sentido amplio, es lo que significa ser hombre o mujer, o también, masculino o femenino, y cómo define este hecho las oportunidades, los papeles, las responsabilidades y las relaciones entre las personas, dentro de una comunidad u organización.

Moser (1989) sostiene que las mujeres realizan tres funciones en la sociedad: reproductivo, productivo y gestión de la comunidad, pero durante mucho tiempo sólo la función reproductiva se ha destacado, porque las mujeres se han visto sobre todo como amas de casa. Ellas han sido marginadas por la desigualdad de oportunidades económicas y las desigualdades de acceso a los recursos productivos tales como el capital y el control sobre su propio trabajo, y por las diferencias en capital humano que lleva a diferencias en la capacidad administrativa y técnica (Loscocco, Robinson, Hall y Allen, 1991). Esta desigualdad ha llevado al desarrollo de teorías que procuran el entendimiento de estos procesos, como la teoría liberal feminista que considera que las mujeres por sí mismas pueden superar la discriminación y otras dificultades dentro de las organizaciones asociadas con el empleo, (Johnsen y McMahon, 2005). Buscan el cambio apelando a los valores liberales de la igualdad, la libertad y el derecho a elegir. Detrás del concepto de feminismo liberal se encuentra el supuesto implícito de que las mujeres y los hombres son iguales si se les conceden las mismas oportunidades (Orser et al., 2010; Fischer, Reuber y Dyke, 1993). Esta teoría no reconoce las diferencias inherentes al género, mientras que la teoría social feminista postula que los hombres y las mujeres están sujetos a procesos de socialización con base en su sexo y que esta condición los hace diferentes (Fischer et al., 1993). Por lo tanto hombres y mujeres tienen formas de pensar y de actuar diferentes pero igual de eficaces y válidas. Sin embargo, estos procesos de socialización ponen a las mujeres en desventaja frente a los hombres a la hora de ingresar al mercado laboral o iniciar a operar su propio negocio (Jones y Tullous, 2002).

En los últimos años, el ingreso de la mujer a la fuerza laboral, ha generado cambios significativos en la estructura de la fuerza laboral y su organización, sobre todo en la búsqueda del derecho de igualdad en oportunidades. Sin embargo, en los diferentes ámbitos empresariales aún se evidencia la persistencia de la desigualdad entre hombres y mujeres, en su ocupación, niveles de responsabilidad y acceso a cargos directivos (Carter et al., 2001, Watson, 2002, Ramos, Barbera y Sarrió, 2003; Ramos, 2005; Cuadrados y Morales, 2007; Fernández et al., 2010; Castaño, Martín, Vázquez y Martínez, 2010, Fuentes y Sánchez, 2010, Torres \& Pau, 2011). La concentración de las mujeres en determinados sectores y puestos de trabajo sigue estando muy marcada, ocupando las mujeres, por lo general, en empleos que son considerados como "no estratégicos" (Fernández et al., 2010; Scheinet, Mueller, Lituchy y Liu, 1996).

Lo anterior se constata con la existencia del denominado "techo de cristal" un término utilizado para designar una barrera invisible que impide a las mujeres cualificadas alcanzar puestos de mayor nivel dentro de las organizaciones y mayores niveles de responsabilidad. Estudios como los de Cuadrado y Morales (2007) y Castaño et al. (2010) muestran que ni la mayor participación de las mujeres en el mercado laboral, ni las medidas adoptadas tendientes a la igualdad, ni la formación académica recibida, han generado un aumento proporcional de la representación femenina en puestos directivos, poniendo de manifiesto que persiste la situación de desventaja de las mujeres en este ámbito. Esta situación de injusticia social ha dado lugar a la denominada perspectiva de género, que centra su análisis en las relaciones de género en la actividad laboral $y$, más concretamente, en la escasa participación de las mujeres en la toma de decisiones y en la ocupación de las posiciones más elevadas (Fernández et al., 2010).

\section{Estudios empíricos previos}

A continuación se presenta una serie de estudios empíricos que ha buscado examinar las diferencias en la gestión que existen en las organizaciones de acuerdo con el género del gerente. Autores como Powell y Eddleston (2011) plantean que los hombres 
y mujeres gestionan los negocios de forma diferente, por lo que caracterizar la gestión de las empresas de acuerdo con el género basándonos en diferentes variables como el nivel educativo, el poco conocimiento en gestión y técnica administrativa, la falta de acceso a mercados, el escaso nivel de riesgo, el tipo de actividad a la que se enfrentan, entre otros, nos permitiría aprender sobre la gestión de las empresas y así poder potenciar las capacidades y actitudes que tienen los gerentes.

Género y tamaño organizacional. Cliff (1998) realiza un estudio donde trata de explicar el tamaño por lo general más pequeño de las empresas dirigidas por mujeres, a través de la actitud que se tiene hacia el crecimiento; para ello, compara el desempeño de las firmas de acuerdo con el género del gerente y concluyen que las empresas dirigidas por mujeres tienen un menor crecimiento y presentan un menor tamaño, generando un problema en el sostenimiento de la empresa en el mercado. Otras evidencias como las de Danes et al., 2007 y Díaz y Jiménez, 2010, señalan también estas diferencias. Huffman, Cohen y Pearlman, (2010) examinan las fuentes de desigualdad de género en las organizaciones, con el objetivo de medir cómo el tamaño de la organización afecta la integración laboral de género a través del tiempo. Encuentran que el tamaño de la organización afecta a la segregación de género, porque existen mayores posibilidades de que las mujeres lleguen a los cargos directivos en empresas de mayor tamaño. Orser et al. (2010), basados en la teoría del proceso de internacionalización de las pequeñas y medianas empresas y los argumentos de la teoría feminista tratan de explicar las diferencias dadas en las exportaciones de acuerdo con el género del propietario de la empresa. Para ello realiza una encuesta a las Pymes canadienses obteniendo respuesta de 8112 empresas. Cuando se introduce la variable categórica género se encuentra que las empresas dirigidas por mujeres son más pequeñas y tienen una orientación menor al crecimiento.

De acuerdo con los estudios empíricos previos, mostrados antes se plantea la siguiente hipótesis:

Hipótesis 1. Las empresas gestionadas por mujeres son de menor tamaño.
Género y edad organizacional. Díaz y Jiménez (2010) plantean desde una perspectiva teórica que las empresas dirigidas por mujeres afrontan ciertas desventajas estructurales como ser empresas con una menor dimensión y una menor antigüedad. Estas desventajas estructurales implican menor disponibilidad de capital humano, social y financiero y en cuanto a la antigüedad de la empresa un menor desarrollo del aprendizaje sobre el comportamiento del mercado. Por lo anterior, se plantea:

Hipótesis 2. Las empresas gestionadas por mujeres son más jóvenes.

Género, sector y nivel tecnológico. Al Marzouqi y Forster (2011) ponen de manifiesto que la mujer no tiene una buena representación en el sector de la tecnología de la información porque no han tenido acceso a la educación en carreras que están orientadas en áreas de la ciencia, la tecnología, la ingeniería y las matemáticas, lo que ha hecho más difícil el manejo de las tecnologías y el uso de las mismas en las actividades desarrolladas; además plantean que las mujeres que ingresan al sector se retiran rápidamente por las exigencias de tiempo y la jornada requerida en estas áreas. Moore, Griffiths, Richardson y Adam (2008) plantea que es un sector donde existe una sub-representación de la mujer y que es probable que no cambie rápidamente en el futuro, porque es más fácil que una mujer salga del sector a que ingrese al mismo. Galyani (2010), plantea que el área de la tecnología de la información es un área predominantemente masculina, porque los hombres y las mujeres tienen diferentes actitudes hacia la tecnología y le dan diferente uso, además la tecnología está asociada con un nivel mayor de riesgo que predomina más en los hombres que en las mujeres. De Luis et al. (2007), plantea que hay una menor representación femenina en la dirección de actividades tecnológicas por la menor participación de la mujer en las carreras tecnológicas y los puestos laborales a los que de ella se acceden. Lo anterior lleva a pensar que las mujeres son menos propensas a la utilización de la tecnología en el desarrollo de su actividad productiva. De Luis et al. (2007) muestran en sus resultados que hay una escasa representación femenina en los cargos de consejos administrativos y de alta dirección en el sector industrial y tecnológico y una mayor representación en el sector orientado a servicios. Para ello utilizan datos recogidos en la publicación "Las 2000 mayores 
empresas españolas" en el período 20002004. Sin embargo, Fernández et al. (2010) señalan que no hay diferencia significativa en relación con los sectores donde se desarrolla la actividad directiva de la mujer. Díaz y Jiménez (2010) abordan su estudio en el sector servicio, porque es donde hay mayor participación de la mujer. Por todo ello, se plantea que:

Hipótesis 3. Las empresas gestionadas por mujeres son mayoritariamente del sector servicios y presentan un menor nivel tecnológico.

Género y carácter familiar. Powell y Eddleston (2011) a partir de la teoría de las funciones de género, realizan una investigación empírica que argumenta que la labor sinérgica que hacen las mujeres en el trabajo y la familia les permite obtener mayores beneficios para el negocio familiar que a los hombres, obteniendo resultados que apoyan esta noción. Concluyen que las mujeres son más capaces de extraer los beneficios empresariales en las empresas familiares. Mateos, Gimeno y Escot, (2010) desarrollan un estudio donde analizan la desigualdad en la promoción de hombres y mujeres y su participación en los consejos administrativos de las 1000 empresas españolas de mayor tamaño de acuerdo con el ingreso de explotación. Los resultados muestran indicios de discriminación hacia la mujer y evidencia que las mujeres alcanzan estos niveles mayormente en las empresas familiares y cooperativas. Knörr (2011) desarrolla un estudio cualitativo que busca determinar por qué las mujeres en los últimos tiempos han decidido desarrollar su propia empresa. Plantea que se han incrementado los negocios donde la propietaria directora es mujer, debido a que es una mejor manera en que las mujeres pueden acceder a niveles más altos en la administración de los negocios y señala que la decisión la toman con base en la experiencia alcanzada en su carrera profesional. Así, podría plantearse que:

Hipótesis 4. Las empresas gestionadas por mujeres son mayoritariamente de carácter familiar.

Género y nivel de formación. Fernández et al. (2010) con el objetivo de contribuir al principio de igualdad de oportunidades en el acceso de la mujer a los cargos directivos, realizan un estudio en 816 Pymes de la Comunidad Autónoma de Andalucía, estudiando si existen diferencias significativas en variables como la edad, la formación, el grado de participación familiar y el sector de actividad con base en el género. Se evidencia que existen diferencias significativas en el grado de formación que poseen los directivos de las Pymes, porque aunque las mujeres tienen niveles de educación altos aún no llegan a los cargos directivos. Justo (2007) plantea que aunque no hay diferencias de género en cuanto al nivel de educación, el campo de educación si puede hacer la diferencia de la mujer en el trabajo y en el proceso de emprendimiento, debido a que sus títulos universitarios están más en el área de las humanidades que en los negocios o las ingenierías u otras disciplinas, encontrándose que el nivel educativo interactúa con el género y el nivel de emprendimiento. Otros estudios como los de Chirwa (2008) y Swinney et al. (2006) señalan que el nivel de educación afecta la relación entre el género y el desempeño de las organizaciones y sus resultados muestran que las mujeres presentan menores niveles de educación. Con lo anterior, se plantea que:

Hipótesis 5. Las empresas gestionadas por mujeres presentan menor nivel de formación.

Género y rendimiento. Driga y Prior (2010) encuentran que el desempeño económico de las empresas dirigidas por mujeres es diferente de las dirigidas por hombres, considerando la posición de partida de las empresas en cuanto a la cifra de capital y al número de empleados. Para ello utilizan la información contable de una muestra de 4450 empresas manufactureras españolas. Los resultados indican diferencias significativas entre las condiciones iniciales de las empresas dirigidas por mujeres y las dirigidas por hombres, aunque las empresas dirigidas por mujeres son más pequeñas (probablemente por la cantidad de recursos que emplean en el arranque) son tan eficaces como las dirigidas por los hombres. Chirwa (2008) estudia el efecto del género en el rendimiento de las micro y pequeñas empresas dirigidas por mujeres y por hombres en el sector no agrícola de Malawi, utilizando datos de encuestas nacionales. Se emplean como indicadores de desempeño, el margen de ganancia y el crecimiento en el empleo, y se realizan tres análisis, el primero general que incluye la variable género, el segundo análisis solo con las empresas propiedad de las mujeres y el tercero con las empresas propiedad de los hombres. El modelo general prueba que el desempeño de las empresas propiedad de mujeres es peor que el desempeño de las empresas propiedad de hombres. Otro estudio realizado por Danes et al. (2007) se centró en la relación moderada entre 
el género de los propietarios de las empresas familiares y el desempeño de las organizaciones con base en las prácticas administrativas y los ingresos brutos. Para ello, utilizaron los datos de la "National Family Business Panel" de Estados Unidos. Los resultados muestran que el género impacta el ingreso bruto de las empresas de manera significativa, encontrando que las empresas de mujeres tienen ingresos brutos inferiores respecto a las empresas propiedad de los hombres. Otros estudios también tienden a apoyar la opinión de que las empresas de propiedad femenina tienen resultados menores que las de propiedad de hombres en términos de ingresos por ventas, valor de activos, márgenes de ganancia y la probabilidad de supervivencia (Loscocco et al., 1991; Carter, Anderson y Shaw, 2001).

Adicionalmente, Charlo y Nuñez (2011) en su estudio analizan las repercusiones económicas que se pueden derivar de la participación de la mujer en los procesos de toma de decisiones de alto nivel en las empresas del sector financiero que cotizan en el mercado español de capitales y concluyen que la presencia de mujeres en la jerarquía directiva y en los consejos de administración de las empresas estudiadas no supone una disminución en los resultados empresariales, por lo que no se entiende por qué las mujeres no acceden a esos puestos en igualdad de condiciones que sus homólogos masculinos. Con base en esto, se plantea:

Hipótesis 6. Las empresas gestionadas por mujeres presentan menor desempeño.

\section{Metodología}

\subsection{Muestra}

Se utilizó una muestra de 600 Pyme de la Región de Murcia, España, y la información procede de la base de datos del Barómetro Económico de la Pyme, que elabora el Instituto de Fomento de la Región de Murcia. La técnica de recogida de información empleada fue la encuesta personal, utilizándose como soporte un cuestionario autoadministrado dirigido al gerente de la empresa. Se adjunta el cuestionario en el anexo. Se realizaron contrastes de control en el proceso de elaboración de la encuesta. El trabajo de campo se inició en marzo y finalizó en septiembre de 2011.

Se emplearon los principios del muestreo estratificado en poblaciones finitas. La población de Pymes en la Región de Murcia es de 95.636. Los criterios utilizados para la estratificación deben estar correlacionados con las variables objeto de estudio teniendo en cuenta que la ganancia en precisión es, en general, decreciente al aumentar el número de estratos. Por ello, el número de criterios y estratos deben ser moderados y congruentes con el máximo tamaño de muestra con el que se pueda trabajar. En este sentido, la población de empresas ha sido segmentada por actividad. El número de empresas en cada uno de los estratos construidos se ha obtenido a partir de la información del Directorio Central de Empresas del INE y de los registros del Régimen General de la Seguridad Social. En la muestra no se incluyen empresas de menos de 5 trabajadores. El tamaño muestral fue determinado para lograr que el margen de error máximo para la estimación de una proporción (frecuencia relativa de respuesta en un punto específico de una pregunta, supuesto de mayor incertidumbre $p=q=0,5$ ) fuese inferior a 0,05 puntos con un nivel de confianza del $95 \%$.

\subsection{Variable dependiente}

\section{Género}

El término "género" significa ser hombre o mujer. En el trabajo de Drigar y Prior (2010) lo refieren al sexo del propietario del negocio, hombre o mujer, mientras que autores como Loscocco et al. (1991), Díaz y Jiménez (2010), entre otros, lo expresan en relación con el sexo del gerente. Para la investigación se utilizó el concepto de género en relación con el sexo del gerente. Siendo una variable categórica que toma el valor 1 cuando la empresa es gestionada por mujer y o cuando es por hombre. En la Tabla 1 se muestra la composición de la muestra según el género del gerente.

Tabla 1. Composición de la muestra según el

tipo de empresa

\begin{tabular}{|c|c|c|}
\hline & Empresas & $(\%)$ \\
\hline $\mathrm{E}^{\mathrm{a}}$ gestionadas por mujeres & 56 & 9,3 \\
\hline $\mathrm{E}^{\mathrm{a}}$ gestionadas por hombres & 544 & 90,6 \\
\hline Total & 600 & 100 \\
\hline
\end{tabular}

\subsection{Variables independientes (de caracterización)}

\section{Tamaño}

El tamaño de la organización es un indicador de crecimiento y desarrollo de las mismas, también de las características necesarias en su administración y se ha asociado con el género del gerente. 
Se puede medir de maneras diferentes; Cliff (1998) lo mide por el éxito del negocio y plantea que las mujeres prefieren no expandirse o crecer, mientras que Orser et al. (2010) lo mide por el número de empleados, por el volumen en ventas y el número de activos y plantea que las empresas gestionadas por mujeres son más pequeñas. En esta investigación el tamaño se mide a partir de dos variables continuas que identifican (1) el nivel de ventas en el año 2010 y (2) el número de empleados en el año 2010. Estas escalas se han trasformado al logaritmo neperiano.

\section{Edad}

La edad o antigüedad de la empresa es un indicador de su capacidad de adaptación al mercado y de su sostenibilidad. Díaz y Jiménez (2010) y Justo (2007) miden la edad por el número de años de creación y la considera como variable de control que caracteriza a las organizaciones. En esta investigación la edad de la empresa se mide a partir de la fecha de constitución, considerando la variable continua número de años en funcionamiento de la empresa hasta la actualidad.

\section{Sector de actividad}

El sector hace referencia a la parte de la economía en las que se agrupan diferentes actividades productivas de la región o país. En esta investigación las empresas se clasificaron en tres sectores, industrial, construcción y servicios. Para el análisis de los datos se define como una variable categórica que toma el valor 1 cuando es del sector servicios y o cuando es del sector industria o construcción. De Luis et al. (2007), define los sectores con base en fomento de la producción en España (Díaz y Jiménez, 2010), y utilizan la rama del sector servicios, porque en España se ha multiplicado por tres su tamaño en la última década.

\section{Carácter familiar de la empresa}

El carácter familiar es el indicador que mide si el grupo familiar posee más del $50 \%$ de la empresa y por tanto si se tiene un control mayoritario sobre la misma. Mateos et al. (2010) para definir una empresa como familiar ha utilizado la dimensión de propiedad y poder y considera una empresa como familiar cuando varios miembros (al menos dos) de una misma familia ocupan cargos en el consejo de administración y/o una parte significativa de las acciones de la empresa es poseída por una misma familia. En esta investigación el carácter familiar de la empresa se identifica a partir de una variable dicotómica que tiene valor 1 cuando la mayoría de la propiedad de la empresa se encuentra en manos de una familia, y valor o en contrario.

\section{Formación del gerente}

La formación del gerente es el grado de escolaridad máximo que ha alcanzado. Justo (2007) utiliza en su modelo el nivel de educación propuesto por el proyecto GEM que define cinco niveles: sin estudios, educación primaria, educación secundaria, estudios medios y estudios superiores. Otros autores como Danes et al. (2007) miden el nivel de educación por el número de años de escolaridad. En esta investigación la formación del gerente se considera a partir de una variable dicotómica que toma valor 1 si el gerente tiene formación universitaria, y valor o en el resto de casos.

\section{Posición tecnológica}

Hace referencia al desarrollo y uso de la tecnología dentro de las organizaciones. Autores como Galyani (2010), Appelbaum et al. (2011) muestran que ésta es un área predominantemente masculina, y que hombres y mujeres tienen diferentes actitudes hacia la tecnología y por lo tanto le dan un diferente uso. En esta investigación la orientación tecnológica de la empresa se ha medido a través del "enfoque del párrafo", para averiguar la percepción que tiene la empresa de su propia tecnología, siguiendo la metodología de AECA (2005). Las distintas categorías de nivel tecnológico son: (a) Posición tecnológica fuerte: la empresa utiliza tecnología desarrollada por ella misma, intentando alcanzar mejores resultados que los competidores; (b) Posición tecnológica buena: la empresa utiliza tecnología adquirida que la posiciona delante de la competencia; (c) Posición tecnológica sostenible: la tecnología usada por la empresa es similar a la de los competidores. La empresa solo invierte en nueva tecnología una vez que la competencia alcanzan buenos resultados; (d) Posición tecnológica débil: los competidores tienen una tecnología más eficiente o moderna que la de la compañía. Para introducir esta información en el presente análisis, se define variables dummy que toma por ejemplo valor 1 cuando la empresa goza de una posición tecnológica débil y valor o para el resto de los casos.

\section{Rendimiento}

El rendimiento es la capacidad empresarial de mostrar resultados en diferentes dimensiones organizacionales. Algunos autores como Chirwa 
(2008) mide el desempeño a través de dos indicadores, el margen de beneficio y el crecimiento en el empleo. Danes et al. (2007) y Díaz y Jiménez (2010), miden el desempeño a través de una medida financiera como son los ingresos brutos. Charlo y Nuñez (2011) mide el éxito empresarial a través de tres indicadores como el margen sobre las ventas, la rentabilidad económica (ROI) y la rentabilidad del accionista (ROE). En esta investigación, el rendimiento de la empresa se ha medido a partir del modelo propuesto por Quinn y Rohrbaugh (1983). Estos autores identifican cuatro modelos de rendimiento: (1) Modelo de procesos internos: se centra en el control interno de la empresa, dando importancia a la comunicación de la información y considerando la estabilidad y el control como los principales objetivos; (2) Modelo de sistema abierto: descansa sobre la importancia de la flexibilidad externa de la empresa, siendo los principales objetivos de la empresa el crecimiento, los recursos y el apoyo externo; (3) Modelo racional: está relacionado con el control desde un punto de vista externo, centrándose en criterios de eficiencia y productividad; y (4) Modelo de relaciones humanas: presta especial atención a la flexibilidad de la organización desde un punto de vista interno, con el objetivo de que los recursos humanos de la empresa se desarrollen dentro de la organización. Para medir estos modelos, se han utilizado 12 puntos en la encuesta (3 para cada modelo), a través de un escala likert de 5 puntos. Para cada modelo se ha calculado la media de la valoración obtenida en cada punto asociado con el modelo. También se ha construido una variable de rendimiento global como la media de los 12 puntos, con un rango teórico de 1 a 5 . Además, para determinar la fiabilidad del instrumento de medición en cada modelo se utilizó el alfa de Cronbach (Tabla 2). Para corroborar la validez del concepto se llevó a cabo un análisis factorial en el que tanto las cargas factoriales como el coeficiente KMO y la prueba de esfericidad de Bartlett arrojan resultados suficientes para validar el indicador de rendimiento, presentado en la Tabla 2.

\section{Tabla 2. Variable de rendimiento}

Indique cuál ha sido la evolución de los siguientes aspectos en su empresa en los dos últimos años: (1= Muy desfavorable, $5=$ Muy favorable)

\begin{tabular}{l|l}
\hline Modelo de & - Calidad del producto/servicio \\
\hline procesos & - Eficiencia de los procesos operativos internos \\
\hline internos & - Organización de las tareas del personal
\end{tabular}

\begin{tabular}{l|l} 
Modelo de & - Satisfacción de los clientes \\
\hline sistema & - Rapidez de adaptación a las necesidades de los mercados \\
\hline abierto & - Imagen de la empresa y de sus productos/servicios
\end{tabular}

Modelo racional

Modelo de relaciones humanas
- Incremento de la cuota de mercado

- Incremento de la rentabilidad

- Incremento de la productividad

- Motivación/satisfacción de los trabajadores

- Reducción de la rotación de personal (abandono voluntario trabajadores)

-Reducción del absentismo laboral
Fiabilidad

Cronbach $a=0,803$

Factorial: 1 factor

Explained variance: $71,9 \%$ Sig. Bartlett: 0,000 KMO: 0,687

Cronbach $a=0.759$

Factorial: 1 factor

Explained variance: $67,6 \%$

Sig. Bartlett: 0,000

KMO: 0,695

Cronbach $a=0,853$

Factorial: 1 factor

Explained variance: $77,3 \%$

Sig. Bartlett: 0,000

KMO: 0,710

Cronbach $a=0,760$

Factorial: 1 factor

Explained variance: $67,6 \%$

Sig. Bartlett: 0,000

KMO: 0,629

\section{Análisis de resultados}

\subsection{Análisis descriptivo de las varia- bles caracterizadoras}

Las variables caracterizadoras continuas muestran que el promedio de empleados de las empresas es de 30,6 con un volumen de ventas de 4'235,326 euros y una antigüedad de 21,02 años. La variable rendimiento medida en una escala de 1 a 5 muestra que los procesos internos tienen un valor promedio de 3,75; el índice de sistema abierto de 3,85; el índice racional de 3,01; el índice de relaciones humanas de 3,43 y el índice de rendimiento global 3,51. Las variables dicotómicas indican que $44,6 \%$ de las empresas pertenecen al sector servicios, $67,3 \%$ son de carácter familiar, $37,8 \%$ tienen gerente con formación universitaria y $22,2 \%$ tienen posición tecnológica débil. En la Tabla 3, se presentan los resultados descriptivos de las variables caracterizadoras. 
Tabla 3. Descriptivos de las variables caracterizadoras

\begin{tabular}{|c|c|c|c|}
\hline $\begin{array}{l}\text { Variables } \\
\text { continuas }\end{array}$ & Media & \multicolumn{2}{|c|}{$\begin{array}{l}\text { Desviación } \\
\text { típica }\end{array}$} \\
\hline $\begin{array}{l}\text { Número medio } \\
\text { de empleados } 2010\end{array}$ & 30,6 & \multicolumn{2}{|c|}{189,6} \\
\hline Ventas 2010 (euros) & $4.235 \cdot 326$ & \multicolumn{2}{|c|}{18.631 .874} \\
\hline Antigüedad & 21,02 & \multicolumn{2}{|l|}{14,5} \\
\hline \multicolumn{4}{|c|}{ Variables de rendimiento } \\
\hline $\begin{array}{l}\text { Procesos internos } \\
\text { Sistema abierto } \\
\text { Racional } \\
\text { Relaciones humanas } \\
\text { Rendimiento global }\end{array}$ & $\begin{array}{c}3,7 \\
3,85 \\
3,01 \\
3,43 \\
3,51\end{array}$ & $\begin{array}{l}0,63 \\
0,65 \\
0,84 \\
0,83 \\
0,58\end{array}$ & \\
\hline \multicolumn{3}{|l|}{ Variables dicotómicas } & $\%$ \\
\hline $\begin{array}{l}\% \text { de empresas del sec } \\
\% \text { de empresas de carä } \\
\% \text { de empresas con ger } \\
\% \text { de empresas con po }\end{array}$ & $\begin{array}{l}\text { servicios } \\
\text { er familiar } \\
\text { e con formació } \\
\text { ión tecnológic }\end{array}$ & $\begin{array}{l}\text { versitaria } \\
\text { bil }\end{array}$ & $\begin{array}{l}44,6 \\
67,3 \\
37,8 \\
22,2\end{array}$ \\
\hline
\end{tabular}

\subsection{Análisis univariante}

Con el propósito de lograr una mayor compresión de los datos, la metodología aplicada se enfoca en determinar si las diferencias observadas entre los dos grupos de interés (hombres y mujeres) son suficientes para aseverar que estos factores tienen una incidencia significativa en el comportamiento diferenciado de los grupos y por lo tanto caracterizan el comportamiento de uno de ellos. En consecuencia, las pruebas estadísticas a realizar se orientan hacia el contraste de la diferencia observada en las medidas de posición central de los indicadores elegidos.
Los contrastes utilizados para el análisis de las variables continuas han sido, el contraste de la t de Student para dos poblaciones independientes, asumiendo varianzas iguales o distintas dependiendo de la prueba de Levene como prueba paramétrica, y el contraste de Mann-Witney, como prueba no paramétrica. En el análisis de las variables categóricas se utilizan tablas de contingencia a las que se les ha aplicado el contraste de $X^{2}$ de Pearson con el fin de valorar si dos variables se encuentran relacionadas. Se ha intentado utilizar tablas $2 X_{2}$ para obtener resultados más potentes, realizando en estos casos la corrección por continuidad de Yates sobre la fórmula de $X^{2}$ de Pearson.

Los resultados presentados en la Tabla 4 muestran que las empresas gestionadas por mujeres tienen un promedio de ventas menor (1'194.373 euros, ventas promedio mujeres y 4'547.886 euros, ventas promedio hombres, significativo al $1 \%$ ) que las gestionadas por hombres. No se encontraron diferencias significativas en la variable número de empleados. Por lo tanto, la hipótesis 1 se cumple parcialmente. Los presentes resultados son coincidentes con los trabajos de Danes et al. (2007) y de Cliff (1998) que muestran cómo las empresas gestionadas por mujeres son más pequeñas, porque prefieren establecer un límite a su crecimiento. Por otra parte y en relación con el número de empleados, este trabajo coincide con el de Driga y Prior (2010) que no encuentran diferencias significativas en el tamaño entre las empresas dirigidas por mujeres o por hombres, cuando este es medido por el número de empleados.

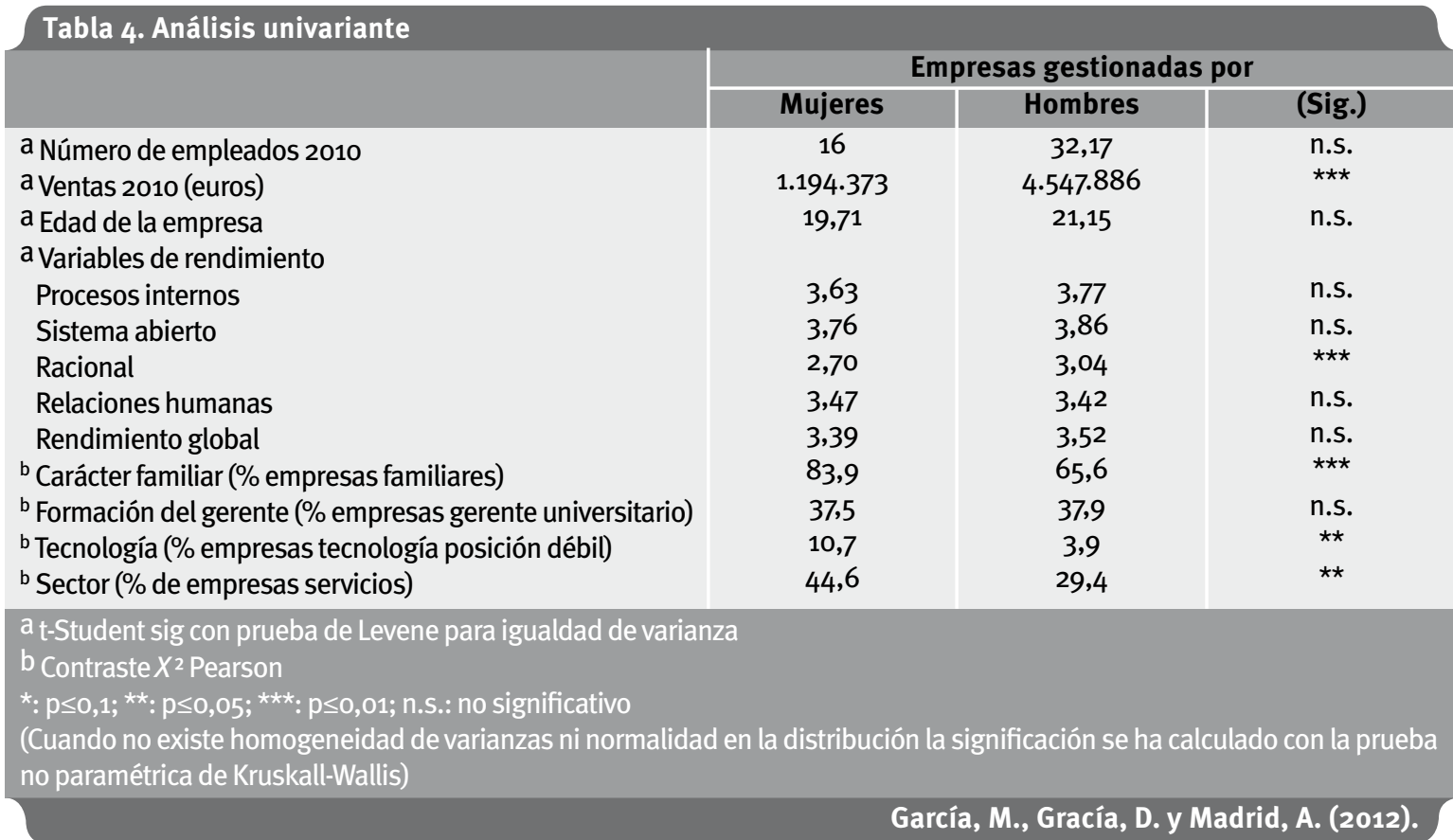


Este estudio evidencia también que las empresas que son gestionadas por mujeres no necesariamente tienen un menor número de años funcionando que las gestionadas por hombres; en este aspecto no se encontró una diferencia significativa entre los dos grupos, y no se cumple con la hipótesis 2. Sin embargo, otros estudios como los de Díaz y Jiménez (2010) y Justo (2007) han puesto de manifiesto el importante papel que juegan la edad y su relación con el género del gerente, pero no muestran un resultado de acuerdo con esta variable que ayude a soportar la misma.

Las empresas gestionadas por mujeres pertenecen en mayor medida al sector servicios (gestionadas por mujeres $44,6 \%$ y $29,4 \%$ por hombres; significativo al $5 \%$ ). Esto da sustento empírico a la hipótesis 3. Estos resultados coinciden con los estudios de De Luis et al. (2007) y Fernández et al. (2010) que plantean que la representación femenina se encuentra mayoritariamente en sectores orientados a los servicios. También se evidencia que las empresas gestionadas por mujeres presentan una posición tecnológica débil en mayor proporción (gestionadas por mujeres $10,7 \%$ y por hombres 3,9\%; significativo al $5 \%$ ). Esto da sustento empírico a la hipótesis 3. Puede deberse como lo plantean Al Marzouqi y Forster (2011) y Galyani (2010) a que la tecnología es una actividad donde predomina el carácter masculino y donde la mujer no ha tenido acceso desde la educación.

Las empresas gestionadas por mujeres en mayor medida son de carácter familiar $(83,9 \%$ de las empresas gestionadas por mujeres son de tipo familiar, mientras que este porcentaje se reduce al $65,6 \%$ en el caso de los hombres y resulta significativo al $1 \%$ ), por lo que se confirma la hipótesis 4. Este resultado es similar al de Fernández et al. (2010) donde obtienen que las mujeres tienen mayor acceso a los niveles directivos cuando la empresa es de carácter familiar o existe una participación familiar mayoritaria. Esto también coincide con Knörr (2011) y Mateos et al. (2010) quienes exponen que hay mayor presencia de las mujeres en lo cargos directivos y consejos en las empresas de carácter familiar, debido a que los negocios familiares ofrecen a las mujeres un lugar más seguro para desarrollar su carrera profesional.

Los resultados muestran que la formación del gerente no es significativa. La hipótesis 5 no tiene por tanto sustento estadístico. Este resultado coincide con el de Fernández et al. (2010) que indican que no hay diferencia significativa entre ser hombre y mujer y el grado de formación del director.

Al examinar la incidencia del género en el rendimiento de la organización se encuentra que las empresas gestionadas por mujeres no presentan diferencias significativas en el rendimiento de procesos internos, sistemas abiertos y relaciones humanas, tampoco en la variable de rendimiento global. Se evidencia diferencia significativa en el rendimiento racional donde se encuentra que las empresas gestionadas por mujeres tienen un menor nivel de rendimiento que las gestionadas por hombres. Por lo tanto, la hipótesis 6 se cumple parcialmente. Estudios como el de Danes et al. (2007) muestran que las mujeres obtienen menos ingresos brutos que los hombres y por lo tanto tienen un menor desempeño. También Swinney et al. (2006) y Chirwa (2008) muestran en sus resultados que el género afecta de forma significativa el desempeño de la organización.

\subsection{Análisis multivariante}

Una vez analizadas las diferencias de comportamiento entre los dos grupos de interés en cuanto a las variables explicativas consideradas de forma individual, se determinará el efecto conjunto de las mismas. Para ello se estima regresiones logísticas bivariantes por el método de Wald por pasos sucesivos. En el modelo se incluyó tan sólo aquellas variables que resultaron significativas a nivel univariante. Se utilizó la metodología de la regresión logística porque la variable dependiente es categórica, identificando cuándo una empresa está siendo gestionada por mujeres o por hombres.

$$
\text { Gestor }_{i}=a_{0}+a_{1} \text { Tamaño }_{i}+a_{2} \text { Racional }_{i}+
$$
$a_{3}$ Familiar $_{i}+a_{4}$ Tecnología $_{i}+a_{5}$ Sector $_{i}+\xi i$

Donde, Gestor es una variable categórica que toma valor 1 para identificar a las empresas gestionadas por mujeres, y o gestionadas por hombres. Tamaño es el logaritmo neperiano de las ventas de la empresa i en el año 2010. Racional es una variable continua que está relacionado con el control desde un punto de vista externo, centrándose en criterios de eficiencia y productividad y mide el rendimiento. Familiar es una variable dicotómica que toma valor de 1 cuando la mayoría de la propiedad de la empresa se encuentra en manos de una familia, y valor o en el resto de los casos; Tecnología es una variable dummy que toma valor 1 cuando es posición débil 
y o para el resto de los casos y Sector es una variable categórica que toma valor de 1 cuando es del sector servicio y o para el resto de los casos.

Además, en la construcción del modelo, se consideró la no normalidad de las variables independientes y las características binaria y cualitativa de la variable dependiente. Para determinar la validez del modelo se calculó la prueba de verosimilitud, para encontrar la estimación más probable de los coeficientes, la medida de Hosmer y Lemeshow de ajuste global, el porcentaje global de acierto en la clasificación y la bondad del ajuste a través de los estadísticos de
R2 alternativos de Cox y Snell y el estadístico de Nagelkerke. Las pruebas estadísticas realizadas confirman la validez de los resultados obtenidos.

Los resultados presentados en la Tabla 5 muestran valores significativos en las variables tamaño, sector, carácter familiar de la empresa y la posición tecnológica débil, lo que nos da evidencia empírica de que las empresas gestionadas por mujeres en la región de Murcia (España) se caracterizan por ser más pequeñas, se encuentran en sector de servicios, son por lo general familiares y tienen una posición tecnológica débil.

\begin{tabular}{|l|c|c|c|c|c|}
\hline Tabla 5. Análisis multivariante. Regresión logística & & & \\
\hline \multicolumn{1}{|c|}{ Variables Independientes } & B & E.T & Wald & Sig. & Exp (B) \\
\hline Tamaño & $-0,285$ & 0,112 & 6,449 & 0,011 & 0,752 \\
Sector de actividad & 0,670 & 0,320 & 4,378 & 0,036 & 1,954 \\
Carácter familiar de la empresa & 0,861 & 0,391 & 4,844 & 0,028 & 2,366 \\
Posición tecnológica & 1,187 & 0,578 & 4,214 & 0,040 & 3,279 \\
\hline
\end{tabular}

Variable Dependiente (Dummy): Empresa gestionada por mujeres $=1$, Empresa gestionada por hombre $=0$

Notas: B: coeficientes logísticos, son medidas de los cambios en el ratio de probabilidades, denominado odds ratio en términos logarítmicos. Un coeficiente positivo aumenta la probabilidad, mientras que un valor negativo disminuye la probabilidad predicha. E.T.: error típico. Wald: estadístico de wald. Sig.: nivel de significación. Exp[B): coeficiente exponenciado. La significación estadística del modelo se ha determinado utilizando el valor de la chi-cuadrado siendo significativo [chi-cuadrado: 21,784, sig: 0,000). La prueba de Hosmer-Lemeschow muestra una chi-cuadrado de 12.350 no significativa (sig: 0.1359) lo que significa que el modelo estimado se ajusta correctamente al comportamiento observado de los datos. Como medida de la calidad de ajuste, obtenemos un porcentaje de aciertos de 65,8\%, 2 log likelihood: 297,401. $R^{2}$ de Cox y Snell: ,041., $R^{2}$ de Nagelkerke: 0,090.

Tamaño: Logaritmo neperiano de las ventas de la empresa i en el año 2010; Sector de actividad: variable categórica que toma valor 1 para las empresas del sector servicios, y valor o en el resto de casos. Carácter Familiar de la Empresa: variable dicotómica que toma valor de 1 cuando la mayoría de la propiedad de la empresa se encuentra en manos de una familia, y valor o en el resto de los casos; Posición tecnológica es una variable dummy que toma valor 1 cuando es posición débil y o para el resto de los casos.

\section{Conclusiones}

En este artículo se estudia cómo el género tiene incidencia en el comportamiento de las Pymes de la región de Murcia (España) con base en diferentes variables de interés como tamaño, edad, carácter familiar, nivel de formación del gerente, posición tecnológica, el sector al que pertenece y rendimiento. Los resultados muestran que las empresas que son dirigidas por mujeres se encuentran sobre todo en el sector de servicios ( $44,6 \%)$, tienen una posición tecnológica débil $(10,7 \%)$, tienen menor tamaño (el promedio en ventas de las empresas de mujeres es de 1'194.373 euros, mientras que los hombres tienen un promedio de 4'547.886 euros), y son sobre todo de carácter familiar $(67,3 \%)$. En relación con el rendimiento solo se encuentra una diferencia significativa en el índice de medición de las características racional, donde se plantea que la mujer presenta un menor rendimiento que los hombres. Estos resultados son coincidentes con estudios previos como los de Fernández et al. (2010); Fuentes y Sánchez (2010) y Knörr (2011) que muestran cómo el ingreso de la mujer al mundo laboral aun hoy se ve influido por el papel que la mujer ha jugado en la sociedad, que la ha enmarcado dentro de estereotipos que se refuerzan con datos empíricos como este. Por ejemplo, la variable sector, por las características y valores de las mujeres, ellas tienen mayor preferencia por ubicarse en el sector servicios, o como en el caso del carácter familiar las mujeres tienen mayor probabilidad de ingreso y de desarrollo.

Esta investigación pretende contribuir al desarrollo de estudios de género en la Pyme y específicamente en las pequeñas y medianas empresas en la región de Murcia (España) porque de acuerdo con los resultados se evidencia que 
aun hoy existen muchas limitaciones para el ingreso y desarrollo de las mujeres dentro de las organizaciones; una de estas es su función social y las obligaciones que este le impone y que afecta las percepciones que se tiene de su desempeño, lo que conduce a trabajar en un cambio cultural dentro de las organizaciones que se oriente a brindar mayores oportunidades de desarrollo y crecimiento a la mujer dentro de la misma. Además, conforme con los resultados del estudio permite plantear que las oportunidades para demostrar las capacidades de las mujeres están aun sin cubrir, debido a la reciente inclusión de las mujeres en el mundo empresarial. Por tanto debe ser un imperativo la creación de políticas de recursos humanos donde influya la equidad de género en cargos directivos.

Este no es un estudio concluido, es el inicio de un proceso de investigación que se orientará a identificar cómo el género incide en la cultura organizacional con el objeto de desarrollar estudios empíricos que permitan aportar al desarrollo teórico y práctico del estudio de las organizaciones.

Los resultados de la investigación pueden ser útiles a las autoridades públicas en la formulación de políticas hacia la mujer considerando sus diferentes funciones (madre, esposa, trabajadora, empresaria, entre otras) dentro de la sociedad y los cambios que estos roles han tenido en los últimos tiempos y los retos que de ellos emergen para las organizaciones y así fomentar las prácticas de buen gobierno en las Pymes y no solo en las empresas grandes. También puede ser útil a los directivos de las Pymes para que tengan elementos de análisis en el diseño de estrategias o políticas que den mayor campo de acción a la mujer dentro de las mismas. Finalmente, puede ser útil a las instituciones educativas porque estudios de investigación como estos les permiten diseñar y actualizar sus programas de estudio e impulsar la realización de otros estudios empíricos que profundicen en los estudios de género y sus características de comportamiento con otras variables que inciden en las organizaciones.

Este trabajo no está exento de limitaciones que deben ser tenidas en cuenta para futuros trabajos. Por una parte, son las derivadas de la información geográfica específica de una región española y la representación de las mujeres en la muestra, pues existe un evidente desequilibrio en la misma, porque ésta contiene 544 hombres y sólo 56 mujeres. Aunque esto viene explicado por la teoría del "Techo de cristal" (Torres y Pau, 2011; Cuadrado y Morales, 2007; Ramos, 2005) que muestra como aún hoy en día, existe una cultura que impide el ingreso de la mujer a las organizaciones y más aún a los altos cargos directivos de las mismas. Y por otra parte, la forma de medir la variable género, considerando tan solo si el gerente/propietario es de sexo femenino 0 masculino.

Estudios futuros deberían considerar la diversidad de género como una oportunidad para estudiar los principios de igualdad en las organizaciones y así aportar al buen gobierno de las mismas. Esta variable se podría medir teniendo en cuenta el número de mujeres total en las organizaciones y los niveles jerárquicos a los que pertenecen y poder analizar los efectos en la segregación vertical y horizontal en las organizaciones pequeñas y medianas. También el estudio permite pensar en futuras investigaciones donde se incluyan análisis psicológicos, antropológicos y sociológicos, donde se analice la aceptación de la mujer en los cargos de dirección y la percepción que pueden tener las poblaciones referente al papel que éstas cumplen en aquellos cargos. Esta cuestión surge, porque sólo $9,3 \%$ de la muestra investigada es de género femenino.

\section{Agradecimientos}

Los autores agradecen el apoyo institucional y económico que para este trabajo han recibido de la Universidad del Valle en Colombia y la Universidad Politécnica de Cartagena en España, así como de la Asociación Universitaria Iberoamericana de Postgrado (AUIP).

\section{Referencias}

AECA. (2005). Estrategia e innovación de la Pyme industrial en España, estudios empíricos. Madrid, España: Asociación Española de Contabilidad y Administración de Empresas.

Al Marzouqi, A.H. \& Forster, N. (2011). An exploratory study of the under-representation of emirate women in the United Arab Emirates' information technology sector. Equality Diversity and Inclusion: An International Journal, 30, (pp. 544-562).

Appelbaum, S.H., Asham, N. \& Argheyd, K. (2011). Is the glass ceiling cracked in infor- 
mation technology? A qualitative analysis: part 1. Industrial and Commercial Training, 43 (6), (pp. 354-361).

Appelbaum, S.H., Asham, N. \& Argheyd, K. (2011). Is the glass ceiling cracked in information technology? A qualitative analysis: part 2. Industrial and Commercial Training, 43 (7), (pp. 451-459).

Ayman, R. \& Korabik, K. (2010). Leadership: why gender and culture matter. American Psychologist, 65 (3), (pp. 157-170).

Bird, B. \& Brush, C. (2002). A gendered perspective on organizational creation. Entrepreneurship Theory and Practice. Spring, 26, (pp. 41-65).

Brush, C. (1992). Research on women business owners: past trends, a new perspective and future directions. Entrepreneurship Theory and Practice. Summer, 16, (pp. 5-30).

Carter, S., Anderson, S. \& Shaw, E. (2001). Women's business ownership: a review of the academic, popular and internet literature. In: Annual Review of Progress in Entrepreneurship (2000 2001). Brussels, Belgium: European Foundation for Management Development (pp. 66-157).

Castaño, C., Martín, J., Vázquez, S. \& Martínez, J.L. (2010). Female executives and the Glass Ceiling in Spain. International Labour Review, 149, (pp-333-360).

Charlo, M.J. \& Núñez, M. (2011). La eficiencia en el sector financiero en España: un enfoque de género. Cuadernos de Administración, Universidad Javeriana, 24 (42), (pp. 147-164).

Cheung, F.M. \& Halpern, D.F. (2010). Women at the top. Powerful leaders define success as work + family in a culture of gender. American Psychologist, 65 (3), (pp. 182-193).

Chirwa, E.W. (2008). Effects of gender on the performance of micro and small enterprises in Malawi. Development Southern Africa, 25 (3), (pp. 347-362).

Cliff, J.E. (1998). Does one size fit all? Exploring the relationship between attitudes towards growth, gender, and business size. Journal of Business Venturing, 13, (pp. 523-542).

Cuadrado, M. y Morales, J.F. (2007). Algunas claves sobre el techo de cristal en las organizaciones. Revista de Psicología del Trabajo y de las Organizaciones, 23 (2), (pp. 183-202).

Danes, S.M., Stafford, K. \& Loy, J.T.C. (2007). Family business performance: the effects of gender and management. Journal of $\mathrm{Bu}$ siness Research, 6o (10), (pp. 1058-1069).

De-Anca, C. y Aragon, S. (2007). La mujer directiva en España: catalizadores e inhibidores en las decisiones de trayectoria profesional. Revista Latinoamericana de Administración, 38, (pp. 45-63).

De-Luis, P., Sanchéz, A., Pérez, M. y Vela, M. J. (2007). La diversidad de género en la alta dirección de las mayores empresas españolas. Investigaciones Europeas de Dirección y Economía de la Empresas, 13, (pp. 33-53).

Díaz, MC. y Jiménez, J.J. (2010). Recursos y resultados de las pequeñas empresas: nuevas perspectivas del efecto género. Cuadernos de Economía y Dirección de la Empresa, 42, (pp. 151-175).

Driga, 0. \& Prior, D. (2010). Start-Up conditions and the performance of women - and men - controlled businesses in manufacturing industries. Revista de Contabilidad - Spanish Accounting Review, 13 (1), (pp. 89-124).

Eagly, A.H. (2005) Achieving relational authenticity in leadership: Does gender matter? The Leadership Quarterly, 16, (pp. 459-474).

Escandón, D.M. y Arias, A. (2011). Factores que componen la competitividad de las empresas creadas por mujeres y las relaciones entre ellos. Cuadernos de Administración, Universidad Javeriana, 24 (42), (pp. 165181).

Fernández, F., López, M., Maeztu, I. y Martín, A. (2010). El techo de cristal en las pequeñas y medianas empresas. Revista de Estudios Empresariales, (1), (pp. 231-247). 
Fischer, E.M., Reuber, R.A., \& Dyke, L.S. (1993). A theoretical overview and extension of research on sex, gender, and entrepreneurship. Journal of Business Venturing, 8 (2), (pp. 151-168).

Fuentes, F.J. y Sánchez, S.M. (2010). Análisis del perfil emprendedor: una perspectiva de género. Estudios de Economía Aplicada, 28 (3), (pp. 1-28).

Gallego, I, García, I.M. \& Rodríguez, L. (2010). The influence of gender diversity on corporate performance. Revista de Contabilidad-Spanish Accounting Review, 13 (1), (pp. 55-88).

Galyani, G. (2010). Information technology and gender gap: toward a global view. The Electronic Library, 28 (5), (pp. 722-733).

Huffman, M.L., Cohen, P.N. \& Pearlman, J. (2010). Engendering change: organizational dynamics and workplace gender desegregation. Administrative Science Quarterly, 55 (2), (pp. 255-277).

Inanova, D. \& Inanova, M. (2011). Gender effects on risk-taking of entrepreneurs: evidence from Bulgaria. International Journal of Entrepreneurial Behaviour \& Research, 17 (3), (pp. 272-295).

Johnsen, G. \& McMahon, R. (2005). Ownermanager gender, financial performance and business growth amongst SMEs from Australia's business longitudinal survey. International Small Business Journal, 23 (2), (pp. 115-140).

Jones, K. \& Tullous, R. (2002). Behaviours of pre-venture entrepreneurs and perceptions of their financial needs. Journal of Small Business Management, 40 (3), (pp. 233-248).

Justo, R. (2007). La influencia del género en el fracaso empresarial: una aplicación al caso de España. Revista de Empresa, (20), (pp. 52-64).

Knörr, H. (2011). From top management to entrepreneurship: wome's next move? International Journal of Manpower, 32 (1), (pp. 99-116).

Lamas, M. (1994). Cuerpo: diferencia sexual y género. Debate Feminista, n. 10, (pp. 3-31).
Lee, J. (2005). Perception of women managers in Singapore: a media analysis. Asia Pacific Business Review, 11 (2), (pp. 233-250).

López, M.P., Gómez, G. y Betancourt, J.B. (2011). Factores que influyen en la participación de la mujer en cargos directivos y órganos de gobierno de la empresa familiar colombiana. Cuadernos de Administración, Universidad Javeriana, 24 (42), (pp. 253-274).

Loscocco, K., Robinson, J., Hall R. \& Allen J. (1991). Gender and small business success: an inquiry into women's relative disadvantage, Social Forces, 70, (pp. 65-85).

Mateos. R., Gimeno, R. y Escot, L. (2010). Discriminación en consejos de administración: análisis e implicaciones económicas. Revista de Economía Aplicada, 18 (53), (pp. 131-162).

Moore, K., Griffiths, M., Richardson, H. \& Adam, A. (2008). Gendered futures? Women, the it workplace and stories of the future. Gender, Work and Organization, 15, (pp. 523-542).

Moser, C. (1989). Gender planning in the third world: meeting practical and strategic gender needs. World Development, 17 (11), (1799-1825).

Orser, B., Spence, M., Riding, A. \& Carrington, C.A. (2010). Gender and export propensity. Entrepreneurship Theory and Practice, 34 (5), (pp. 933-958).

Powell. G.N. \& Eddleston, K.A. (2011). Workfamily enrichment and entrepreneurial success: do female entrepreneurs benefit most? The Academy of Management, 32 (3), (pp. 747-76o).

Quinn, R. \& Rohrbaugh, J. (1983). A spatial model of effectiveness criteria: towards a competing values approach to organizational analysis. Management Science, 29 (3), (pp. 363-377).

Ramos, A. (2005). Mujeres directivas: un valor en alza para las organizaciones laborales. Cuadernos de Geografía. (78), (pp. 191-214).

Ramos, A., Barberá, E. y Sarrió, M. (2003). Mujeres directivas, espacio de poder y relaciones de género. Anuario de Psicología, 34 (2), (pp. 267-278). 
Sánchez-Apellániz, M. (1997). Mujeres, dirección, y cultura organizacional. Centro de Investigaciones Sociológicas. Madrid, España: Centro de Investigaciones Sociologicas.

Schein, V., Mueller, R., Lituchy, T. \& Liu, J. (1996). Hink manager-thinkmale: a global phenomenon. Journal of Organizational Behaviour, 17, (pp. 33-41).

Swinney, J.L., Runyan, R.C. \& Huddleston, P. (2006). Differences in reported firm performance by gender: does industry matter? Journal of Developmental Entrepreneurship, 11 (2), (pp. 99-115).

Talmud, I. \& Izraeli, D.N. (1999). The relationship between gender and performance issues of concern to directors: correlates or institution. Journal of Organizational Behavior, 20, (pp. 459-474).

Torres, 0. y Pau, B. (2011). "Techo de cristal” y "suelo pegajoso". La situación de la mujer en los sistemas alemán y español de ciencia y tecnología. Revista Iberoamericana de Ciencia, Tecnología y Sociedad, 6 (18), (pp. 35-59).

Watson, J. (2002). Comparing the performance of male- and female- controlled businesses: relating outputs to inputs. Entrepreneurship: Theory and Practice, 22, (pp. 91-100).

Yordanova, D. (2011). The effects of gender on entrepreneurship in Bulgaria: an empirical study. International Journal of Management, 28 (1), (pp. 289-305). 